\title{
Minimum-Width Double-Strip and Parallelogram Annulus
}

\author{
Sang Won Bae \\ Division of Computer Science and Engineering, Kyonggi University, Suwon, Korea \\ swbae@kgu.ac.kr
}

\begin{abstract}
In this paper, we study the problem of computing a minimum-width double-strip or parallelogram annulus that encloses a given set of $n$ points in the plane. A double-strip is a closed region in the plane whose boundary consists of four parallel lines and a parallelogram annulus is a closed region between two edge-parallel parallelograms. We present several first algorithms for these problems. Among them are $O\left(n^{2}\right)$ and $O\left(n^{3} \log n\right)$-time algorithms that compute a minimum-width double-strip and parallelogram annulus, respectively, when their orientations can be freely chosen.
\end{abstract}

2012 ACM Subject Classification Theory of computation $\rightarrow$ Computational geometry

Keywords and phrases geometric covering, parallelogram annulus, two-line center, double-strip

Digital Object Identifier 10.4230/LIPIcs.ISAAC.2019.25

Related Version https://arxiv.org/abs/1911.07504

Funding Sang Won Bae: Supported by Basic Science Research Program through the National Research Foundation of Korea (NRF) funded by the Ministry of Education (2018R1D1A1B07042755).

\section{Introduction}

The minimum-width annulus problem asks to find an annulus of a certain shape with the minimum width that encloses a given set $P$ of $n$ points in the plane. An annulus informally depicts a ring-shaped region in the plane. As the most natural and classical example, a circular annulus is defined to be the region between two concentric circles. If one wants to find a circle that best fits an input point set $P$, then her problem can be solved by finding out a minimum-width circular annulus that encloses $P$. After early results on the circular annulus problem [13], the currently best algorithm that computes a minimum-width circular annulus that encloses $n$ input points takes $O\left(n^{\frac{3}{2}+\epsilon}\right)$ time [3,4] for any $\epsilon>0$. Analogously, such a problem of matching a point set into a closed curve class can be formulated into the minimum-width annulus problem for annuli of different shapes.

Along with applications not only to the points-to-curve matching problem but also to other types of facility location, the minimum-width annulus problem has been extensively studied for recent years, with a variety of variations and extensions. Abellanas et al. [1] considered minimum-width rectangular annuli that are axis-parallel, and presented two algorithms taking $O(n)$ or $O(n \log n)$ time: one minimizes the width over rectangular annuli with arbitrary aspect ratio and the other does over rectangular annuli with a prescribed aspect ratio, respectively. Gluchshenko et al. [9] presented an $O(n \log n)$-time algorithm that computes a minimum-width axis-parallel square annulus, and proved a matching lower bound, while the second algorithm by Abellanas et al. can do the same in the same time bound. If one considers rectangular or square annuli in arbitrary orientation, the problem becomes more difficult. Mukherjee et al. [12] presented an $O\left(n^{2} \log n\right)$-time algorithm that computes a minimum-width rectangular annulus in arbitrary orientation and arbitrary aspect ratio. The author [5] showed that a minimum-width square annulus in arbitrary orientation can be computed in $O\left(n^{3} \log n\right)$ time, and recently improved it to $O\left(n^{3}\right)$ time [6]. 
In this paper, we consider a more generalized shape, namely, parallelograms, and annuli based on them in fixed or arbitrary orientation, which have at least one more degree of freedom than square and rectangular annuli have. More precisely, we define a parallelogram annulus as a closed region between two edge-parallel parallelograms, and address the problem of computing a minimum-width parallelogram annulus that encloses the input point set $P$. (See Figure 1(c).) We consider several restricted cases of the problem about two orientations of sides of parallelogram annuli. Our main results are summarized as follows:

(1) When both orientations for sides are fixed, a minimum-width parallelogram annulus that encloses $P$ can be computed in $O(n)$ time.

(2) When one orientation for sides is fixed and the other can be chosen arbitrarily, a minimum-width parallelogram annulus that encloses $P$ can be computed in $O\left(n^{2}\right)$ time.

(3) A minimum-width parallelogram annulus that encloses $P$ over all pairs of orientations can be computed in $O\left(n^{3} \log n\right)$ time.

To obtain these algorithms for the problem, we also introduce another geometric optimization problem, called the minimum-width double-strip problem, which asks to compute a double-strip of minimum width that encloses $P$. A double-strip is defined to be the union of two parallel strips, where a strip is the region between two parallel lines in the plane. (See Figure 1(b).) We show that this new problem is closely related to the parallelogram annulus problem. The minimum-width double-strip problem has its own interest as a special case of the two-line center problem, in which one wants to find two strips, possibly being non-parallel, that encloses $P$ and minimizes the width of the wider strip. After the first sub-cubic algorithm is presented by Agarwal and Sharir [2], the currently best algorithm for the two-line center problem takes $O\left(n^{2} \log ^{2} n\right)$ time $[8,11]$.

To our best knowledge, however, no nontrivial result on the double-strip problem is known in the literature. In this paper, we obtain the following algorithmic results:

(4) A minimum-width double-strip that encloses $P$ over all orientations can be computed in $O\left(n^{2}\right)$ time.

(5) We also consider a constrained version of the problem in which a subset $Q \subseteq P$ with $k=|Q|$ is given and one wants to find a minimum-width double-strip enclosing $Q$ such that all points of $P$ should lie in between its outer boundary lines. We show that this can be solved in $O(n \log n+k n)$ time.

(6) We further address some online and offline versions of the dynamic constrained doublestrip problem under insertions and/or deletions of a point on the subset $Q$ to enclose.

The rest of the paper is organized as follows: Section 2 introduces necessary definitions and preliminaries. Section 3 is devoted to solve the minimum-width double-strip problem and present an $O\left(n^{2}\right)$ time algorithm, which is generalized to the constrained double-strip problem in Section 4. The minimum-width parallelogram annulus problem is finally discussed and solved in Section 5 .

Omitted proofs and additional figures will be provided in a full version.

\section{Preliminaries}

In this section, we introduce definitions of necessary concepts and preliminaries for further discussion. For any subset $A \subseteq \mathbb{R}^{2}$ of the plane $\mathbb{R}^{2}$, its boundary and interior are denoted by $\partial A$ and $\operatorname{int} A$, respectively.

For two parallel lines $\ell$ and $\ell^{\prime}$ in the plane $\mathbb{R}^{2}$, the distance between $\ell$ and $\ell^{\prime}$ denotes the length of any line segment that is orthogonal to $\ell$ and $\ell^{\prime}$ and have endpoints one on $\ell$ and the other on $\ell^{\prime}$. A strip is a closed region bounded by two parallel lines in the plane. For any 


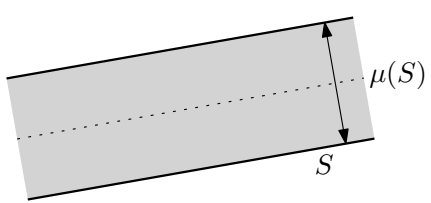

(a)

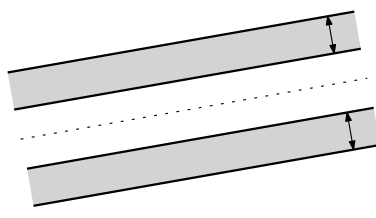

(b)

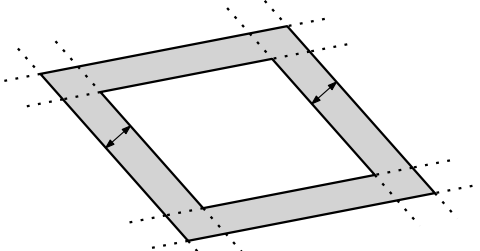

(c)

Figure 1 Illustrations to (a) a strip $S$ and its middle line $\mu(S)$, (b) a double-strip, and (c) a parallelogram annulus. The arrows depict the width of each shape.

strip $S$, its width $w(S)$ is the distance between its bounding lines, and its middle line $\mu(S)$ is the line parallel to its bounding lines such that the distance between $\mu(S)$ and each of the bounding lines is exactly half the width $w(S)$ of $S$. See Figure 1(a).

A double-strip is the union of two disjoint parallel strips of equal width, or equivalently, is a closed region obtained by a strip $S$ subtracted by the interior of another strip $S^{\prime}$ such that $\mu(S)=\mu\left(S^{\prime}\right)$ and $S^{\prime} \subseteq S$. For any double-strip defined by two strips $S$ and $S^{\prime}$ in this way, $S$ is called its outer strip and $S^{\prime}$ its inner strip. The width of such a double-strip $D$, denoted by $w(D)$, is defined to be half the difference of the widths of $S$ and $S^{\prime}$, that is, $w(D)=\left(w(S)-w\left(S^{\prime}\right)\right) / 2$. See Figure 1(b).

A parallelogram is a quadrilateral that is the intersection of two non-parallel strips. We define a parallelogram annulus to be a parallelogram $R$ with a parallelogram hole $R^{\prime}$, analogously as a circular annulus is a circle with a circular hole. Here, we add a condition that the outer and inner parallelograms $R$ and $R^{\prime}$ should be side-wise parallel. There are several ways to define such a parallelogram annulus, among which we introduce the following definition. A parallelogram annulus $A$ is defined by two double-strips $D_{1}$ and $D_{2}$ as follows: 1. The outer parallelogram $R$ of $A$ is the intersection of the outer strips of $D_{1}$ and $D_{2}$.

2. The inner parallelogram $R^{\prime}$ of $A$ is the intersection of the inner strips of $D_{1}$ and $D_{2}$.

3. The parallelogram annulus $A$ is the closed region between $R$ and $R^{\prime}$, that is, $A=R \backslash \operatorname{int} R^{\prime}$.

4. The width of $A$, denoted by $w(A)$, is taken to be the bigger one between the widths of $D_{1}$ and $D_{2}$, that is, $w(A)=\max \left\{w\left(D_{1}\right), w\left(D_{2}\right)\right\}$.

See Figure 1(c) for an illustration.

The main purpose of this paper is to solve the minimum-width parallelogram annulus problem in which we are given a set $P$ of points in the plane and want to find a parallelogram annulus of minimum width that encloses $P$. As discussed above, a parallelogram annulus is closely related to strips and double-strips. The minimum-width double-strip problem asks to find a double-strip of minimum width that encloses $P$ in fixed or arbitrary orientation.

The orientation of a line or line segment $\ell$ in the plane is a value $\theta \in[-\pi / 2, \pi / 2)^{1}$ such that the rotated copy of the $x$-axis by $\theta$ counter-clockwise is parallel to $\ell$. If the orientation of a line or line segment is $\theta$, then we say that the line or line segment is $\theta$-aligned. A strip or a double-strip is also called $\theta$-aligned for some $\theta \in[-\pi / 2, \pi / 2)$ if its bounding lines are $\theta$-aligned. A parallelogram or a parallelogram annulus is $(\theta, \phi)$-aligned if it is defined by two double-strips that are $\theta$-aligned and $\phi$-aligned, respectively.

For any two points $p, q \in \mathbb{R}^{2}$, let $\overline{p q}$ denote the line segment joining $p$ and $q$, and $|\overline{p q}|$ denote the Euclidean length of $\overline{p q}$. We will often discuss the strip defined by two parallel lines through $p$ and $q$, and its width. Let $\sigma_{\theta}(p, q)$ be the width of the strip defined by two $\theta$-aligned lines through $p$ and $q$, respectively. It is not difficult to see that $\sigma_{\theta}(p, q)=|\overline{p q}| \cdot\left|\sin \left(\theta-\theta_{p q}\right)\right|$, where $\theta_{p q} \in[-\pi / 2, \pi / 2)$ denotes the orientation of $\overline{p q}$. See Figure 2(a).

\footnotetext{
1 The orientation $\theta$ is indeed of period $\pi$. In this paper we choose $[-\pi / 2, \pi / 2)$ for the orientation domain.
} 


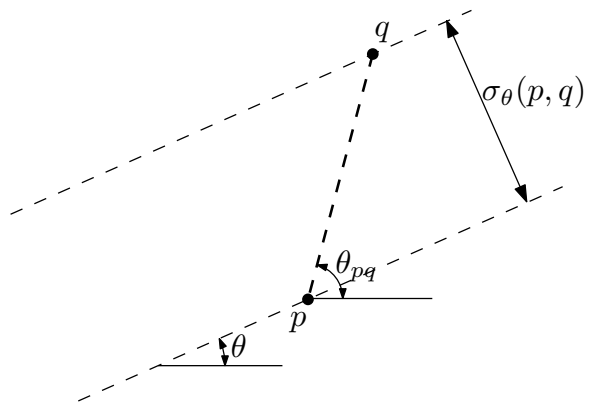

(a)

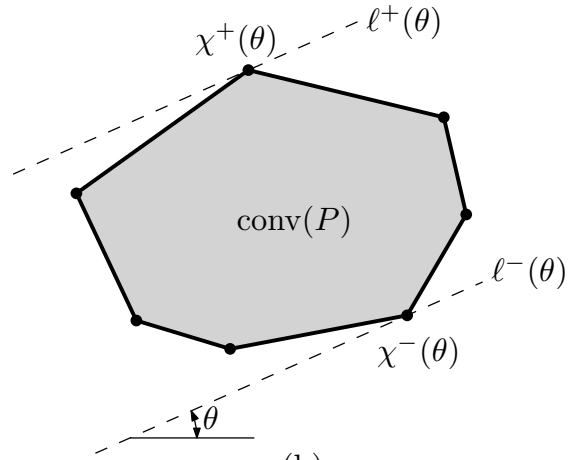

(b)

Figure 2 (a) The $\theta$-aligned strip defined by $p$ and $q$, and its width $\sigma_{\theta}(p, q)$. (b) The antipodal pair $\left(\chi^{+}(\theta), \chi^{-}(\theta)\right)$ of $P$ for orientation $\theta \in[-\pi / 2, \pi / 2)$.

A single-variate function of a particular form $a \sin (\theta+b)$ for some constants $a, b \in \mathbb{R}$ with $a \neq 0$ is called sinusoidal function (of period $2 \pi$ ). Obviously, the equation $a \sin (\theta+b)=0$ has at most one zero over $\theta \in[-\pi / 2, \pi / 2)$. The following property of sinusoidal functions is well known and easily derived.

- Lemma 1. The sum of two sinusoidal functions is also sinusoidal. Therefore, the graphs of two sinusoidal functions cross at most once over $[-\pi / 2, \pi / 2)$.

Note that, taking $\theta \in[-\pi / 2, \pi / 2)$ as a variable, the function $\sigma_{\theta}(p, q)$ for fixed points $p, q \in \mathbb{R}^{2}$ is piecewise sinusoidal with at most one breakpoint.

Let $P$ be a finite set of points in $\mathbb{R}^{2}$, and let $\operatorname{conv}(P)$ be its convex hull. The corners of $\operatorname{conv}(P)$ are called extreme of $P$. For each $\theta \in[-\pi / 2, \pi / 2)$, let $S(\theta)$ be the minimum-width $\theta$-aligned strip enclosing $P$. Then, the two lines that define $S(\theta)$ pass through two extreme points of $P$, one on each. More precisely, let $\ell^{+}(\theta)$ and $\ell^{-}(\theta)$ be the two lines defining $S(\theta)$ such that $\ell^{+}(\theta)$ lies above $\ell^{-}(\theta)$ if $\theta \neq-\pi / 2$, or $\ell^{+}(\theta)$ lies to the right of $\ell^{-}(\theta)$ if $\theta=-\pi / 2$. There must be an extreme point of $P$ on each of the two lines $\ell^{+}(\theta)$ and $\ell^{-}(\theta)$, denoted by $\chi^{+}(\theta)$ and $\chi^{-}(\theta)$. If there are two or more such points of $P$, then we choose the last one in the counter-clockwise order along the boundary of $\operatorname{conv}(P)$. The width of $S(\theta)$ is equal to $\sigma_{\theta}\left(\chi^{+}(\theta), \chi^{-}(\theta)\right)$.

For each $\theta \in[-\pi / 2, \pi / 2)$, the pair $\left(\chi^{+}(\theta), \chi^{-}(\theta)\right)$ is called antipodal. It is known that there are at most $O(n)$ different antipodal pairs by Toussaint [14]. See Figure 2(b). Starting from $\theta=-\pi / 2$, imagine the motion of the two lines $\ell^{+}(\theta)$ and $\ell^{-}(\theta)$ as $\theta$ continuously increases. Then, the antipodal pair $\left(\chi^{+}(\theta), \chi^{-}(\theta)\right)$ for $\theta$ only changes when one of the two lines contains an edge of $\operatorname{conv}(P)$. In this way, the orientation domain $[-\pi / 2, \pi / 2)$ is decomposed into maximal intervals $I$ such that $\left(\chi^{+}(\theta), \chi^{-}(\theta)\right)$ remains the same in $I$.

\section{Minimum-Width Double-Strips}

In this section, we address the problem of computing a minimum-width double-strip that encloses a given set $P$ of $n$ points in $\mathbb{R}^{2}$.

We start with the problem in a given orientation $\theta \in[-\pi / 2, \pi / 2)$. Let $w(\theta)$ be the minimum possible width of a $\theta$-aligned double-strip enclosing $P$. The following observation can be obtained by a simple geometric argument. 
- Observation 2. For each $\theta \in[-\pi / 2, \pi / 2)$, there exists a minimum-width $\theta$-aligned doublestrip $D(\theta)$ enclosing $P$ whose outer strip is $S(\theta)$ and inner strip is $S^{\prime}(\theta)$, where

- $S(\theta)$ is the minimum-width $\theta$-aligned strip enclosing $P$, and

- $S^{\prime}(\theta)$ is the maximum-width $\theta$-aligned strip such that its interior is empty of any point in $P$ and $\mu\left(S^{\prime}(\theta)\right)=\mu(S(\theta))$.

Proof. Let $D$ be a minimum-width $\theta$-aligned double-strip enclosing $P$. Let $S_{1}$ and $S_{2}$ be the two strips such that $D=S_{1} \cup S_{2}$. If the boundary of $S_{1}$ does not contain an extreme point of $P$, then we can slide $S_{1}$ inwards until its boundary hits an extreme point of $P$. Let $S_{1}^{\prime}$ be the resulting strip after this sliding process. Then, we have $S_{1} \cap P \subset S_{1}^{\prime}$. In the same way, we slide $S_{2}$ until the boundary of $S_{2}$ hits an extreme point of $P$, and let $S_{2}^{\prime}$ be the resulting strip. Then, it holds that $S_{2} \cap P \subset S_{2}^{\prime}$. As a result, $P \subset\left(S_{1}^{\prime} \cup S_{2}^{\prime}\right)$ since $P \subset\left(S_{1} \cup S_{2}\right)$. Note that the outer strip of the double-strip $D^{\prime}:=S_{1}^{\prime} \cup S_{2}^{\prime}$ is exactly $S(\theta)$, the minimum-width $\theta$-aligned strip enclosing $P$.

Now, let $S^{\prime}$ be the inner strip of $D^{\prime}$. By definition, we have $\mu(S(\theta))=\mu\left(S^{\prime}\right)$. Suppose that the inner strip $S^{\prime}$ of $D^{\prime}$ is not equal to $S^{\prime}(\theta)$, the maximum-width $\theta$-aligned strip such that its interior is empty of any point in $P$ and $\mu\left(S^{\prime}(\theta)\right)=\mu(S(\theta))$. Then, the boundary of $S^{\prime}$ does not contain any point of $P$. Hence, the width of $S^{\prime}(\theta)$ is strictly larger than the width of $S^{\prime}$, a contradiction that $D^{\prime}$ is of minimum width. Therefore, the inner strip of $D^{\prime}$ should be $S^{\prime}(\theta)$.

We focus on finding the minimum-width double-strip $D(\theta)$ described in Observation 2 . The outer strip $S(\theta)$ of $D(\theta)$ is determined by $\ell^{+}(\theta)$ and $\ell^{-}(\theta)$ on which the two extreme points $\chi^{+}(\theta)$ and $\chi^{-}(\theta)$ lie. For $p \in P$, let $d_{p}(\theta):=\min \left\{\sigma_{\theta}\left(p, \chi^{+}(\theta)\right), \sigma_{\theta}\left(p, \chi^{-}(\theta)\right)\right\}$. Then, the width $w(\theta)$ of $D(\theta)$ in orientation $\theta$ is determined by

$$
w(\theta)=\max _{p \in P} d_{p}(\theta) .
$$

It is not difficult to see that $w(\theta)$ can be evaluated in $O(n)$ time for a given $\theta \in[-\pi / 2, \pi / 2)$.

Theorem 3. Given a set $P$ of $n$ points and an orientation $\theta \in[-\pi / 2, \pi / 2)$, a minimumwidth $\theta$-aligned double-strip enclosing $P$ and its width $w(\theta)$ can be computed in $O(n)$ time.

Proof. In this proof, we describe our algorithm that computes $D(\theta)$. Given $\theta$, one can compute the two extreme points $\chi^{+}(\theta)$ and $\chi^{-}(\theta)$ in $O(n)$ time by computing the minimum and maximum among the inner products of

$$
(\cos (\theta+\pi / 2)), \sin (\theta+\pi / 2))
$$

and all points in $P$ as vectors. The antipodal pair also determines the outer strip $S(\theta)$.

After identifying $\chi^{+}(\theta)$ and $\chi^{-}(\theta)$, the value of $d_{p}(\theta)$ for each $p \in P$ can be computed in $O(1)$ time. Hence, $w(\theta)=\max _{p \in P} d_{p}(\theta)$ can be found in additional $O(n)$ time. This determines the inner strip $S^{\prime}(\theta)$.

Next, we turn to finding a minimum-width double-strip over all orientations. This is equivalent to computing the minimum value of $w(\theta)$ over $\theta \in[-\pi / 2, \pi / 2)$, denoted by $w^{*}$. Let $\theta^{*}$ be an optimal orientation such that $w\left(\theta^{*}\right)=w^{*}$. Consider the corresponding double-strip $D\left(\theta^{*}\right)$ whose outer strip is $S\left(\theta^{*}\right)$ and inner strip is $S^{\prime}\left(\theta^{*}\right)$, as described in Observation 2. We then observe the following for the minimum-width double-strip $D\left(\theta^{*}\right)$ enclosing $P$.

Lemma 4. Let $\theta^{*}$ be an orientation such that $w\left(\theta^{*}\right)=w^{*}$. Then, either

(a) three extreme points of $P$ lie on the boundary of $S\left(\theta^{*}\right)$, or

(b) two points of $P$ lie on the boundary of $S^{\prime}\left(\theta^{*}\right)$. 


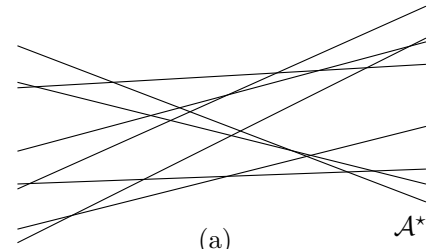

(a)

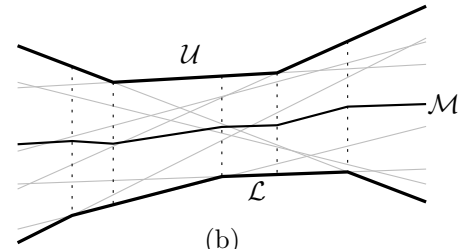

(b)

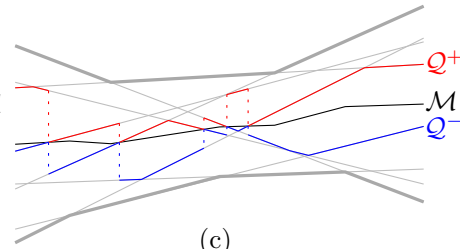

(c)

Figure 3 (a) The arrangement $\mathcal{A}^{\star}$ of lines in $P^{\star}$. (b) The upper envelope $\mathcal{U}$, the lower envelope $\mathcal{L}$, and $\mathcal{M}$ of $\mathcal{A}^{\star}$. (c) $\mathcal{Q}^{+}$and $\mathcal{Q}^{-}$depicted by red and blue chains, respectively.

Regard $d_{p}(\theta)$ as a function of $\theta$ in domain $[-\pi / 2, \pi / 2)$. This function depends on the antipodal pair of extreme points $\left(\chi^{+}(\theta), \chi^{-}(\theta)\right)$ for $\theta$. Since there are $O(n)$ antipodal pairs [14], the function $d_{p}$ for each $p \in P$ is piecewise sinusoidal with $O(n)$ breakpoints. The width function $w(\theta)$ is the upper envelope of the $n$ functions $d_{p}(\theta)$ for $p \in P$, consisting of $O\left(n^{2}\right)$ sinusoidal curves in total. Thus, we can compute the minimum width $w^{*}=$ $\min _{\theta \in[-\pi / 2, \pi / 2)} w(\theta)$ by computing the upper envelope of these $O\left(n^{2}\right)$ sinusoidal curves and finding a lowest point in the envelope. Since any two sinusoidal curves cross at most once by Lemma 1 , this can be done in $O\left(n^{2} \log n\right)$ time [10]. The width function $w$ is piecewise sinusoidal with $O\left(n^{2} \alpha(n)\right)$ breakpoints, where $\alpha$ denotes the inverse Ackermann function. and its lowest point always occurs at one of the breakpoints by Lemma 4 . Hence, $O\left(n^{2} \log n\right)$ time is sufficient to solve the problem.

In the following, we improve this to $O\left(n^{2}\right)$ time. As observed above, the double-strip $D(\theta)$ of width $w(\theta)$ is determined by its outer strip $S(\theta)$ and inner strip $S^{\prime}(\theta)$. Let $\mu(\theta):=$ $\mu(S(\theta))=\mu\left(S^{\prime}(\theta)\right)$ be the middle line of $D(\theta)$. The middle line $\mu(\theta)$ separates $P$ into two subsets $P^{+}(\theta)$ and $P^{-}(\theta)$, where $P^{+}(\theta)$ is the set of points $p \in P$ lying in the strip defined by $\ell^{+}(\theta)$ and $\mu(\theta)$, and $P^{-}(\theta)=P \backslash P^{+}(\theta)$. Define $q^{+}(\theta) \in P^{+}(\theta)$ to be the closest point to line $\mu(\theta)$ among $P^{+}(\theta)$, and similarly $q^{-}(\theta) \in P^{-}(\theta)$ to be the closest point to $\mu(\theta)$ among $P^{-}(\theta)$. We then observe that the width of $D(\theta)$ is

$$
w(\theta)=\max \left\{\sigma_{\theta}\left(q^{+}(\theta), \chi^{+}(\theta)\right), \sigma_{\theta}\left(q^{-}(\theta), \chi^{-}(\theta)\right)\right\} .
$$

Hence, $D(\theta)$ is completely determined by these four points: $\chi^{+}(\theta), \chi^{-}(\theta), q^{+}(\theta)$, and $q^{-}(\theta)$.

In order to analyze and specify the change of pair $\left(q^{+}(\theta), q^{-}(\theta)\right)$ as $\theta$ continuously increases from $-\pi / 2$ to $\pi / 2$, we adopt an interpretation under a geometric dualization [7, Chapter 8]. We shall call the plane $\mathbb{R}^{2}$ - in which we have discussed objects so far - the primal plane with the $x$ - and $y$-axes. Let $\mathbb{D}$ be another plane, called the dual plane, with $u$ - and $v$-axes that correspond to its horizontal and vertical axes, respectively. Here, we use a standard duality transform ${ }^{\star}$ which maps a point $p=(a, b) \in \mathbb{R}^{2}$ into a line $p^{\star}: v=a u-b \subset \mathbb{D}$ and a non-vertical line $\ell: y=a x-b \subset \mathbb{R}^{2}$ into a point $\ell^{\star}=(a, b) \in \mathbb{D}$. This duality transform is also defined in the reversed way for points and lines in the dual plane $\mathbb{D}$ to be mapped to lines and points in the primal plane $\mathbb{R}^{2}$, so that we have $\left(p^{\star}\right)^{\star}=p$ and $\left(\ell^{\star}\right)^{\star}=\ell$ for any point $p$ and any non-vertical line $\ell$ either in $\mathbb{R}^{2}$ or in $\mathbb{D}$. We say that a geometric object and its image under the duality transform are dual to each other. Note that $p$ lies above (on or below, resp.) $\ell$ if and only if $\ell^{\star}$ lies above (on or below, resp.) $p^{\star}$.

\subsection{Scenes from the dual plane}

Suppose that the input point set $P$ is given in the primal plane $\mathbb{R}^{2}$. Consider the set $P^{\star}:=\left\{p^{\star} \mid p \in P\right\}$ of $n$ lines in the dual plane $\mathbb{D}$ and their arrangement $\mathcal{A}^{\star}:=\mathcal{A}\left(P^{\star}\right)$. See Figure 3 for illustration. Let $\mathcal{U}$ and $\mathcal{L}$ be the upper and lower envelopes in $\mathcal{A}^{\star}$. The 
envelopes $\mathcal{U}$ and $\mathcal{L}$ can also be considered as two functions of $u \in \mathbb{R}$; in this way, $\mathcal{U}(u)$ and $\mathcal{L}(u)$ are the $v$-coordinates in $\mathbb{D}$ of points on $\mathcal{U}$ and $\mathcal{L}$, respectively, at $u \in \mathbb{R}$. Let $\mathcal{M}(u):=(\mathcal{U}(u)+\mathcal{L}(u)) / 2$ be the $v$-coordinates of the midpoint of the vertical segment connecting $\mathcal{L}$ and $\mathcal{U}$ at $u \in \mathbb{R}$. Similarly, we regard $\mathcal{M}$ as the function itself and simultaneously as its graph $\mathcal{M}=\{(u, \mathcal{M}(u)) \mid u \in \mathbb{R}\}$ drawn in $\mathbb{D}$.

As well known, the upper envelope $\mathcal{U}$ corresponds to the lower chain of $\operatorname{conv}(P)$ and the lower envelope $\mathcal{L}$ to its upper chain. More precisely, each vertex of $\mathcal{U}$ and $\mathcal{L}$ is dual to the line supporting an edge of $\operatorname{conv}(P)$. Thus, the total number of vertices of $\mathcal{U}$ and $\mathcal{L}$ is no more than $n=|P|$. Also, observe that the number of vertices of $\mathcal{M}$ is equal to the total number of vertices of $\mathcal{U}$ and $\mathcal{L}$ by definition.

Now, consider the portions of lines in $P^{\star}$ above $\mathcal{M}$ and the lower envelope of those pieces cut by $\mathcal{M}$, denoted by $\mathcal{Q}^{+}$. Analogously, let $\mathcal{Q}^{-}$be the upper envelope of portions of lines in $P^{\star}$ below $\mathcal{M}$. The following observations follow directly from the basic properties of duality.

- Observation 5. For each $\theta \in[-\pi / 2, \pi / 2)$, let $u:=\tan \theta$. Then, the following hold:

(1) The dual $\left(\ell^{+}(\theta)\right)^{\star}$ of the $\theta$-aligned line through $\chi^{+}(\theta)$ is the point $(u, \mathcal{L}(u))$ in $\mathbb{D}$. Similarly, we have $\left(\ell^{-}(\theta)\right)^{\star}=(u, \mathcal{U}(u))$.

(2) The dual $(\mu(\theta))^{\star}$ of the middle line $\mu(\theta)$ is the point $(u, \mathcal{M}(u))$ in $\mathbb{D}$.

(3) The dual of the $\theta$-aligned line through $q^{+}(\theta)$ is the point $\left(u, \mathcal{Q}^{-}(u)\right)$ in $\mathbb{D}$. Similarly, the dual of the $\theta$-aligned line through $q^{-}(\theta)$ is the point $\left(u, \mathcal{Q}^{+}(u)\right)$.

From Observation 5, one would say informally that $\ell^{+}$is dual to $\mathcal{L}, \ell^{-}$to $\mathcal{U}, \mu$ to $\mathcal{M}, q^{+}$to $\mathcal{Q}^{-}$, and $q^{-}$to $\mathcal{Q}^{+}$.

We are ready to describe our algorithm. We first compute the arrangement $\mathcal{A}^{\star}$ in $O\left(n^{2}\right)$ time. The envelopes $\mathcal{U}$ and $\mathcal{L}$ can be traced in $O(n)$ time from $\mathcal{A}^{\star}$, and we also can compute $\mathcal{M}$ in $O(n)$ time. We then compute $\mathcal{Q}^{+}$and $\mathcal{Q}^{-}$.

- Lemma 6. The complexity of $\mathcal{Q}^{+}$and $\mathcal{Q}^{-}$is $O\left(n^{2}\right)$, and we can compute them in $O\left(n^{2}\right)$ time.

By Lemma 6 together with Observation 5, we know that there are $O\left(n^{2}\right)$ changes in pair $\left(q^{+}(\theta), q^{-}(\theta)\right)$ as $\theta$ increases from $-\pi / 2$ to $\pi / 2$. On the other hand, we already know that the antipodal pair $\left(\chi^{+}(\theta), \chi^{-}(\theta)\right)$ changes $O(n)$ times as $\theta$ increases from $-\pi / 2$ to $\pi / 2$. Consequently, there are $O\left(n^{2}\right)$ changes in tuple $\left(\chi^{+}(\theta), \chi^{-}(\theta), q^{+}(\theta), q^{-}(\theta)\right)$, and thus the orientation domain $[-\pi / 2, \pi / 2)$ is decomposed into $O\left(n^{2}\right)$ intervals in each of which the tuple is fixed. For each such interval $I$, we minimize $w(\theta)=\max \left\{\sigma_{\theta}\left(q^{+}(\theta), \chi^{+}(\theta)\right), \sigma_{\theta}\left(q^{-}(\theta), \chi^{-}(\theta)\right)\right\}$ over $\theta \in I$. Since the four points $\chi^{+}(\theta), \chi^{-}(\theta), q^{+}(\theta), q^{-}(\theta)$ are fixed in $I$, the function $w$ on $I$ is the upper envelope of at most four sinusoidal functions by Lemma 1. By Lemma 4, the minimum occurs either (a) at an endpoint of $I$ or (b) when the equality $\sigma_{\theta}\left(q^{+}(\theta), \chi^{+}(\theta)\right)=$ $\sigma_{\theta}\left(q^{-}(\theta), \chi^{-}(\theta)\right)$ holds. Hence, we can minimize $w(\theta)$ over $\theta \in I$ in $O(1)$ time. Since $w^{*}=\min _{I} \min _{\theta \in I} w(\theta)$, we can compute $w^{*}$ by taking the minimum over such intervals $I$.

- Theorem 7. Given a set $P$ of $n$ points in the plane, a minimum-width double-strip enclosing $P$ can be computed in $O\left(n^{2}\right)$ time.

\section{Constrained Double-Strip Problem}

In this section, we discuss a constrained version of the minimum-width double-strip problem, called the constrained double-strip problem. The constrained double-strip problem has its own interest, while it can also be used, in particular, to obtain efficient algorithms for the parallelogram annulus problem, which will be discussed in the following section. 
In the constrained double-strip problem, we are given a set $P$ of $n$ points and a subset $Q \subseteq P$ with $k=|Q|$. A $P$-constrained double-strip is a double-strip whose outer strip contains all points in $P$. Then, the problem asks to find a $P$-constrained double-strip of minimum width that encloses subset $Q$.

Analogously to Observation 2, we observe the following for the constrained problem.

- Observation 8. For each $\theta \in[-\pi / 2, \pi / 2)$, there exists a minimum-width $\theta$-aligned $P$ constrained double-strip $D_{Q}(\theta)$ enclosing $Q$ such that its outer strip is $S(\theta)$ and its inner strip is $S_{Q}^{\prime}(\theta)$, where $S_{Q}^{\prime}(\theta)$ is the maximum-width $\theta$-aligned strip such that its interior is empty of any point in $Q$ and $\mu\left(S_{Q}^{\prime}(\theta)\right)=\mu(\theta)$.

Let $w_{Q}(\theta)$ be the width of the minimum-width $P$-constrained double-strip $D_{Q}(\theta)$ enclosing $Q$ described in Observation 8 , and $w_{Q}^{*}:=\min _{\theta \in[-\pi / 2, \pi / 2)} w_{Q}(\theta)$. Hence, we focus on computing the minimum possible width $w_{Q}^{*}$ and its corresponding double-strip $D_{Q}\left(w_{Q}^{*}\right)$. We also redefine $q^{+}(\theta)$ and $q^{-}(\theta)$ to be the closest points to $\mu(\theta)$ among points in $Q$ above and below $\mu(\theta)$, respectively. Then, we have

$$
w_{Q}(\theta)=\max \left\{\sigma_{\theta}\left(q^{+}(\theta), \chi^{+}(\theta)\right), \sigma_{\theta}\left(q^{-}(\theta), \chi^{-}(\theta)\right)\right\} .
$$

The following lemma is analogous to Lemma 4.

- Lemma 9. Let $\theta^{*}$ be an orientation such that $w_{Q}\left(\theta^{*}\right)=w_{Q}^{*}$. Then, either

(a) three extreme points of $P$ lie on the boundary of $S\left(\theta^{*}\right)$, or

(b) two points of $Q$ lie on the boundary of $S_{Q}^{\prime}\left(\theta^{*}\right)$.

To solve the constrained problem, we extend our approach for the unconstrained problem. Define $\mathcal{A}_{Q}^{\star}:=\mathcal{A}\left(Q^{\star} \cup \mathcal{U} \cup \mathcal{L}\right)$ to be the arrangement of $k$ lines in $Q^{\star}=\left\{p^{\star} \mid p \in Q\right\}$ plus the envelopes $\mathcal{U}$ and $\mathcal{L}$ of $\mathcal{A}\left(P^{\star}\right)$. Let $\mathcal{Q}_{Q}^{+}$be the lower envelope of portions of lines in $Q^{\star}$ above $\mathcal{M}$, and $\mathcal{Q}_{Q}^{-}$be the upper envelope of portions of lines in $Q^{\star}$ below $\mathcal{M}$.

Our algorithm for the constrained double-strip problem runs as follows: First, compute the convex hull $\operatorname{conv}(P)$ and extract $\mathcal{U}$ and $\mathcal{L}$ from $\operatorname{conv}(P)$ in $O(n \log n)$ time. Then, we add lines in $Q^{\star}$ incrementally one by one to build $\mathcal{A}_{Q}^{\star}$. Since every line in $Q^{\star}$ lies between $\mathcal{U}$ and $\mathcal{L}$, this takes additional $O\left(k^{2}\right)$ time. Next, we compute $\mathcal{Q}_{Q}^{+}$and $\mathcal{Q}_{Q}^{-}$.

- Lemma 10. The complexity of $\mathcal{Q}_{Q}^{+}$and $\mathcal{Q}_{Q}^{-}$is $O(k n)$, and we can compute them in $O(k n)$ time.

The rest of the algorithm is the same as that described in the previous section. As we increase $\theta \in[-\pi / 2, \pi / 2)$ continuously, there are $O(k n)$ changes in tuple $\left(\chi^{+}(\theta), \chi^{-}(\theta), q^{+}(\theta), q^{-}(\theta)\right)$ by Lemma 10 , and thus the orientation domain $[-\pi / 2, \pi / 2)$ is decomposed into $O(k n)$ intervals in each of which the four points are fixed. For each such interval $I$, we minimize $w_{Q}(\theta)=$ $\max \left\{\sigma_{\theta}\left(q^{+}(\theta), \chi^{+}(\theta)\right), \sigma_{\theta}\left(q^{-}(\theta), \chi^{-}(\theta)\right)\right\}$ over $\theta \in I$. By Lemma 9, the minimum occurs either (a) at an endpoint of $I$ or (b) when the equality $\sigma_{\theta}\left(q^{+}(\theta), \chi^{+}(\theta)\right)=\sigma_{\theta}\left(q^{-}(\theta), \chi^{-}(\theta)\right)$ holds. Hence, we can minimize $w(\theta)$ over $\theta \in I$ in $O(1)$ time, and obtain the following result.

- Theorem 11. Given a set $P$ of $n$ points and a subset $Q \subseteq P$ with $k=|Q|$, a minimum-width $P$-constrained double-strip enclosing $Q$ can be computed in $O(n \log n+k n)$ time.

\subsection{Dynamic maintenance under insertion and deletion}

In the following, we consider a dynamic situation in which a point in $P$ can be inserted into or deleted from $Q$. Our goal is to report a minimum-width $P$-constrained double-strip enclosing $Q$ over all orientations and its width, that is, $w_{Q}^{*}$ and $D_{Q}\left(w_{Q}^{*}\right)$, whenever a change in $Q$ occurs, faster than computing from scratch by Theorem 11 . 


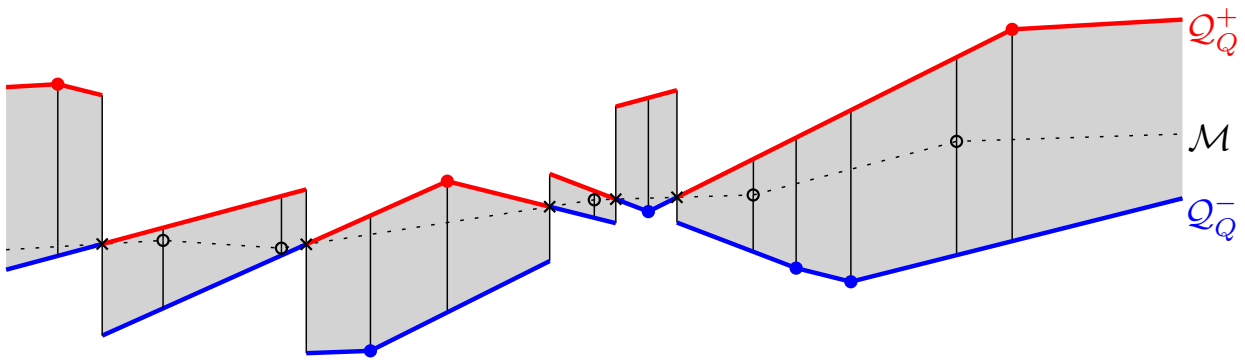

Figure 4 Illustration to the trapezoidal map $\mathcal{T}_{Q}$ with 18 trapezoids. In this example, we take $Q=P$ for input point set $P$, being the same as the one used as in Figure 3. Red and blue dots are vertices of $\mathcal{A}_{Q}^{\star}$ that belong to $\mathcal{Q}_{Q}^{+}$and $\mathcal{Q}_{Q}^{-}$, respectively. Small circles depict the vertices of $\mathcal{M}$ and cross marks are the intersections between $\mathcal{M}$ and any line $p^{\star}$ for $p \in Q$.

For the purpose, we keep the following invariants updated under insertions and deletions: the arrangement $\mathcal{A}_{Q}^{\star}$ and a trapezoidal map $\mathcal{T}_{Q}$. The map $\mathcal{T}_{Q}$ is a vertical trapezoidal decomposition of the region between $\mathcal{Q}_{Q}^{+}$and $\mathcal{Q}_{Q}^{-}$. More precisely, let $\mathcal{Q}_{Q}$ be the region between $\mathcal{Q}_{Q}^{+}$and $\mathcal{Q}_{Q}^{-}$, and $\left\{u_{1}, u_{2}, \ldots, u_{m}\right\}$ be the set of $u$-coordinates of the vertices of $\mathcal{Q}_{Q}^{+}$, $\mathcal{Q}_{Q}^{-}$, and $\mathcal{M}$ such that $u_{1}<u_{2}<\cdots<u_{m}$. By adding into $\mathcal{Q}_{Q}$ a vertical line segment at $u=u_{i}$ for each $i=1,2, \ldots, m$ between two points $\left(u_{i}, \mathcal{Q}_{Q}^{-}\left(u_{i}\right)\right)$ and $\left(u_{i}, \mathcal{Q}_{Q}^{+}\left(u_{i}\right)\right)$, we obtain the trapezoidal decomposition $\mathcal{T}_{Q}$ consisting of $m+1$ trapezoids. For convenience, let $u_{0}:=-\infty$ and $u_{m+1}:=\infty$. The order of trapezoids in $\mathcal{T}_{Q}$ is naturally induced along the $u$-axis in $\mathbb{D}$. The $i$-th trapezoid $\tau$ in $\mathcal{T}_{Q}$ for $i=1, \ldots, m+1$ is bounded by two vertical segments at $u=u_{i-1}$ and $u=u_{i}$, and two segments from $\mathcal{Q}_{Q}^{+}$and $\mathcal{Q}_{Q}^{-}$. See Figure 4 for an illustration. The two segments of $\tau$ from $\mathcal{Q}_{Q}^{+}$and $\mathcal{Q}_{Q}^{-}$are called the ceiling and floor of $\tau$, respectively. Let $U_{\tau} \subset \mathbb{R}$ be an interval consisting of the $u$-coordinates of all points in $\tau$.

At each trapezoid $\tau$, we store four points $\chi_{\tau}^{+}, \chi_{\tau}^{-}, q_{\tau}^{+}, q_{\tau}^{-} \in P$ such that $\chi_{\tau}^{+}=\chi^{+}(\theta)$, $\chi_{\tau}^{-}=\chi^{-}(\theta), q_{\tau}^{+}=q^{+}(\theta)$, and $q_{\tau}^{-}=q^{-}(\theta)$ for all $\theta$ with $\tan \theta \in U_{\tau}$. We also store at $\tau$ the value $w_{\tau}:=\min _{\tan \theta \in U_{\tau}}\left\{\max \left\{\sigma_{\theta}\left(q_{\tau}^{+}, \chi_{\tau}^{+}\right), \sigma_{\theta}\left(q_{\tau}^{-}, \chi_{\tau}^{-}\right)\right\}\right\}$, which can be computed in $O(1)$ time per trapezoid by Lemma 9 .

Note that the union of ceilings of all trapezoids in $\mathcal{T}_{Q}$ forms $\mathcal{Q}_{Q}^{+}$, and the union of their floors forms $\mathcal{Q}_{Q}^{-}$. By Lemma 10, the number $m+1$ of trapezoids in $\mathcal{T}_{Q}$ is $O(|Q| n)$. More importantly, for each $\theta \in[-\pi / 2, \pi / 2)$, there is a unique trapezoid $\tau$ in $\mathcal{T}_{Q}$ such that $\tan \theta$ lies in interval $U_{\tau}$ of $\tau$ and thus we have $w_{Q}(\theta)=\max \left\{\sigma_{\theta}\left(q_{\tau}^{+}, \chi_{\tau}^{+}\right), \sigma_{\theta}\left(q_{\tau}^{-}, \chi_{\tau}^{-}\right)\right\}$. This implies that $\min \left\{w_{Q}(\theta) \mid \tan \theta \in U_{\tau}\right\}=w_{\tau}$, and hence $w_{Q}^{*}=\min _{\tau \in \mathcal{T}_{Q}} w_{\tau}$. Thus, by efficiently maintaining $\mathcal{T}_{Q}$, the problem is reduced to finding the minimum of $w_{\tau}$ over all trapezoids $\tau$ in $\mathcal{T}_{Q}$.

Updating our invariants can be done in $O(n)$ time thanks to the Zone Theorem for the arrangement of lines.

Lemma 12. Let $Q \subseteq P, p \in P$, and $Q^{\prime}$ be either $Q \cup\{p\}$ or $Q \backslash\{p\}$. Then, $\mathcal{A}_{Q^{\prime}}^{\star}$ and $\mathcal{T}_{Q^{\prime}}$ can be obtained in $O(n)$ time, provided the description of $\mathcal{A}_{Q}^{\star}$ and $\mathcal{T}_{Q}$. More specifically, the number of trapezoids in the symmetric difference $\left(\mathcal{T}_{Q^{\prime}} \backslash \mathcal{T}_{Q}\right) \cup\left(\mathcal{T}_{Q} \backslash \mathcal{T}_{Q^{\prime}}\right)$ is $O(n)$, and those trapezoids can be identified in the same time bound.

Now, we are ready to describe our overall algorithm. We assume that we start with an empty set $Q=\emptyset$ and a sequence of insertions and deletions on $Q$ is given. For $Q=\emptyset$, it is easy to initialize $\mathcal{A}_{\emptyset}^{\star}$ and $\mathcal{T}_{\emptyset}$, after computing $\mathcal{U}, \mathcal{L}$, and $\mathcal{M}$ in $O(n \log n)$ time as described above. Namely, $\mathcal{A}_{\emptyset}^{\star}$ is just the union of $\mathcal{U}$ and $\mathcal{L}$, and $\mathcal{T}_{\emptyset}$ can be obtained from the fact that $\mathcal{Q}_{\emptyset}^{+}=\mathcal{U}$ and $\mathcal{Q}_{\emptyset}^{-}=\mathcal{L}$. Whenever an insertion or deletion of a point is given, we update our 
invariants as described in Lemma 12, spending $O(n)$ time. We then report the minimum possible width $w_{Q}^{*}$ for the current subset $Q$ and the corresponding double-strip. Since $\mathcal{T}_{Q}$ consists of $O(|Q| n)$ trapezoids by Lemma 10, a linear scan of $\mathcal{T}_{Q}$ is too costly. We instead use a basic priority queue, such as the binary heap, and conclude the following.

- Theorem 13. Let $P$ be a set of $n$ points, and $Q_{0}=\emptyset, Q_{1}, Q_{2}, \ldots$ be a sequence of subsets of $P$ such that the difference between $Q_{i+1}$ and $Q_{i}$ is only a single point in $P$. Suppose that each $Q_{i}$ is given at time $i$ by its difference from $Q_{i-1}$. Then, whenever $Q_{i}$ is specified for each $i \geq 0$, we can exactly compute a P-constrained double-strip of minimum width $w_{Q_{i}}^{*}$ that encloses $Q_{i}$ in $O(n \log n)$ time.

If one only wants to decide whether or not the minimum possible width $w_{Q}^{*}$ is at least a given target value $w \geq 0$, then the complexity can be reduced as follows.

- Theorem 14. Let $P$ be a set of $n$ points, and $Q_{0}=\emptyset, Q_{1}, Q_{2}, \ldots$ be a sequence of subsets of $P$ such that the difference between $Q_{i+1}$ and $Q_{i}$ is only a single point in $P$. Suppose that each $Q_{i}$ is given at time $i$ by its difference from $Q_{i-1}$. Let $w \geq 0$ be a given fixed real number. Then, after spending $O(n \log n)$ time for preprocessing, whenever $Q_{i}$ is specified for each $i \geq 0$, we can decide whether $w \geq w_{Q_{i}}^{*}$ or $w<w_{Q_{i}}^{*}$ in $O(n)$ time.

\subsection{Offline version under insertions only}

Note that the above theorems give us solutions to the online optimization and decision versions of the $P$-constrained double-strip problem under insertions and deletions. Here, we consider the offline optimization version of the problem under insertions only.

Let $Q=\left\{p_{1}, p_{2}, \ldots, p_{k}\right\} \subseteq P$ be a subset of $P$, and $Q_{i}:=\left\{p_{1}, \ldots, p_{i}\right\}$ for $i=0, \ldots, k$. Suppose that we know $Q_{i}$ for each $i=0, \ldots, k$ for the first time and want to compute a minimum-width $P$-constrained double-strip enclosing $Q_{i}$ for all $i=0, \ldots, k$.

For the purpose, we observe the following.

- Lemma 15. For each $i=0, \ldots, k-1$, it holds that $w_{Q_{i}}^{*}=\min \left\{w_{Q_{i+1}}^{*}, \min _{\tau \in T_{i}} w_{\tau}\right\}$, where $T_{i}:=\mathcal{T}_{Q_{i}} \backslash \mathcal{T}_{Q_{i+1}}$ denotes the set of trapezoids removed from $\mathcal{T}_{Q_{i}}$ by the insertion of $p_{i+1}$.

Lemma 15 suggests computing $w_{Q_{i}}^{*}$ backwards from $i=k$ to $i=0$. By maintaining $\mathcal{T}_{Q_{i}}$ from $i=0$ to $k$ and storing the sets $T_{i}=\mathcal{T}_{Q_{i}} \backslash \mathcal{T}_{Q_{i+1}}$, this can be done in $O(k n)$ time.

More precisely, we first build $\mathcal{A}_{Q_{0}}^{\star}$ and $\mathcal{T}_{Q_{0}}$ as described above in $O(n \log n)$ time. We then insert $p_{i}$ for $i=1, \ldots, k$, one by one, and compute $\mathcal{A}_{Q_{i}}^{\star}$ and $\mathcal{T}_{Q_{i}}$ in $O(n)$ time per insertion by Lemma 12 , but we do not compute the minimum width $w_{Q_{i}}^{*}$ at every insertion. Instead, we collect all trapezoids $\tau$ that have been deleted, that is, the set $T_{i}=\mathcal{T}_{Q_{i}} \backslash \mathcal{T}_{Q_{i+1}}$. Then, we apply Lemma 15 to compute $w_{Q_{i}}^{*}$ for each $i=0, \ldots, k$ and its corresponding double-strip.

We first compute $w_{Q_{k}}^{*}=\min _{\tau \in \mathcal{T}_{Q_{k}}} w_{\tau}$. Then, we iterate $i$ from $k-1$ to 0 , and compute $w_{Q_{i}}^{*}$ based on Lemma 15. Since $\left|T_{i}\right|=O(n)$ for each $i$ by Lemma 12, this takes $O(k n)$ additional time. We thus conclude the following theorem.

- Theorem 16. Let $P$ be a set of $n$ points and $p_{1}, \ldots, p_{k} \in P$ be $k \geq 1$ points in $P$, and let $Q_{i}:=\left\{p_{1}, \ldots, p_{i}\right\}$ for $0 \leq i \leq k$. Then, in time $O(n \log n+k n)$, we can exactly compute $w_{Q_{i}}^{*}$ for all $0 \leq i \leq k$ and corresponding $P$-constrained double-strips of width $w_{Q_{i}}^{*}$ enclosing $Q_{i}$. 


\section{Minimum-Width Parallelogram Annuli}

In this section, we present algorithms that compute a minimum-width parallelogram annulus that encloses a set $P$ of $n$ points in $\mathbb{R}^{2}$. As introduced in Section 2, a parallelogram annulus is defined by two double-strips and its orientation is represented by a pair of parameters $(\theta, \phi)$ with $\theta, \phi \in[-\pi / 2, \pi / 2)$.

Here, we consider several cases depending on how many of the two side orientations, $\theta$ and $\phi$, are fixed or not. The easiest case is certainly when both $\theta$ and $\phi$ are fixed.

- Observation 17. For any $\theta, \phi \in[-\pi / 2, \pi / 2)$, there exists a minimum-width $(\theta, \phi)$-aligned parallelogram annulus that encloses $P$ such that its outer parallelogram $R(\theta, \phi)$ is the intersection of $S(\theta)$ and $S(\phi)$, the minimum-width $\theta$-aligned and $\phi$-aligned strip enclosing $P$, respectively.

The above observation gives us a structural property of an optimal annulus which we should look for, and leads to a linear-time algorithm.

- Theorem 18. Given a set $P$ of $n$ points and $\theta, \phi \in[-\pi / 2, \pi / 2)$, a minimum-with $(\theta, \phi)$ aligned parallelogram annulus that encloses $P$ can be computed in $O(n)$ time.

Proof. Here, we describe an algorithm that finds a minimum-width $(\theta, \phi)$-aligned parallelogram annulus enclosing $P$ whose outer parallelogram is $R(\theta, \phi)=S(\theta) \cap S(\phi)$, as described in Observation 17.

As described in the proof of Theorem 3, we can find the minimum-width $\theta$-aligned strip $S(\theta)$ enclosing $P$ in $O(n)$ time by identifying the corresponding antipodal pair $\left(\chi^{+}(\theta), \chi^{-}(\theta)\right)$. In the same way, we identify the antipodal pair $\left(\chi^{+}(\phi), \chi^{-}(\phi)\right)$ and strip $S(\phi)$.

We then need to find an inner parallelogram $R^{\prime}$ that minimizes the width of the resulting annulus defined by $R(\theta, \phi)$ and $R^{\prime}$. For the purpose, we are done by checking the distance from each $p \in P$ to the boundary of $R$, which is equal to

$$
\min \left\{w_{\theta}\left(p, \chi^{+}(\theta)\right), w_{\theta}\left(p, \chi^{-}(\theta)\right), w_{\phi}\left(p, \chi^{+}(\phi)\right), w_{\phi}\left(p, \chi^{-}(\phi)\right)\right\} .
$$

We can evaluate this in $O(1)$ time for each $p \in P$, so in $O(n)$ total time, and take the maximum over them, denoted by $z$. Since the interior of the inner parallelogram $R^{\prime}$ must avoid all points in $P$, at least one side of $R^{\prime}$ must be $z$ distant from the boundary of $R$. Hence, the minimum width of a $(\theta, \phi)$-aligned parallelogram annulus enclosing $P$ is exactly $z$. The value of $z$ and a corresponding annulus can be computed in $O(n)$ time.

In the following, let $w(\theta, \phi)$ be the smallest among the widths of all $(\theta, \phi)$-aligned parallelogram annuli enclosing $P$.

\subsection{When one side orientation is fixed}

Next, we consider the problem where one side of a resulting annulus should be $\phi$-aligned for a fixed orientation $\phi \in[-\pi / 2, \pi / 2)$ while the other orientation parameter $\theta$ can be chosen arbitrarily. So, in the following, we regard $\phi \in[-\pi / 2, \pi / 2)$ to be fixed. Without loss of generality, we assume that $\phi=0$.

From the definition of a parallelogram annulus $A$, it is defined by two double-strips. In addition, Observation 17 tells us that the two double-strips defining $A$ can be chosen among the $P$-constrained double-trips enclosing a subset of $P$. Hence, for the case where $\phi=0$ is fixed, the problem is reduced to find a best bipartition of $P$ such that one part is covered by a 0 -aligned $P$-constrained double-strip and the other by another $P$-constrained double-strip in any orientation $\theta \in[-\pi / 1, \pi / 2)$. 
We first identify two extreme points $\chi^{+}(0)$ and $\chi^{-}(0)$, and the strip $S(0)$ in $O(n)$ time. Then, sort the points in $P$ in the non-increasing order of the value

$$
d_{p}(0)=\min \left\{\sigma_{0}\left(p, \chi^{+}(0)\right), \sigma_{0}\left(p, \chi^{-}(0)\right)\right\}
$$

for each $p \in P$, which is the distance to the boundary of $S(0)$. Let $p_{1}, p_{2}, \ldots, p_{n-1}, p_{n} \in P$ be this order. Also, let $w_{i}:=d_{p_{i}}(0)=\min \left\{\sigma_{0}\left(p_{i}, \chi^{+}(0)\right), \sigma_{0}\left(p_{i}, \chi^{-}(0)\right)\right\}$ for $i=1, \ldots, n$.

Consider the double-strip $D_{i}$ with width $w_{i}$ and outer strip $S(0)$. The double-strip $D_{1}$ encloses all points of $P$, while $D_{2}$ misses one point $p_{1}$ and $D_{i}$ misses $i-1$ points $p_{1}, \ldots, p_{i-1}$ in general for $i=1, \ldots, n$. This means that there are only $n$ different subsets of $P$ covered by any 0 -aligned double-strip. Thus, to enclose $P$ by a $(\theta, 0)$-aligned parallelogram annulus, the other double-strip with orientation $\theta$ should cover the rest of the points in $P$.

Note that each $D_{i}$ is a minimum-width 0-aligned $P$-constrained double-strip that encloses $\left\{p_{i}, p_{i+1}, \ldots, p_{n}\right\} \subseteq P$. Let $Q_{i}:=\left\{p_{1}, p_{2}, \ldots, p_{i-1}\right\}$ for $i=1, \ldots, n$. If we choose $D_{i}$ for the 0-aligned double-strip, then $P \backslash Q_{i} \subset D_{i}$, so the points in $Q_{i}$ should be covered by the second double-strip that define a parallelogram annulus. Let $D_{i}^{\prime}$ be the minimum-width $P$-constrained double-strip enclosing $Q_{i}$, and let $w_{i}^{\prime}$ be its width. We compute $D_{i}^{\prime}$ and $w_{i}^{\prime}$ for all $i \in\{1, \ldots, n\}$ by applying Theorem 16 in $O\left(n^{2}\right)$ time. What remains is taking the minimum of $\max \left\{w_{i}, w_{i}^{\prime}\right\}$ over $i=1, \ldots, n$.

- Theorem 19. Given a set $P$ of $n$ points and a fixed orientation $\phi \in[-\pi / 2, \pi / 2)$, a $(\theta, \phi)$-aligned parallelogram annulus of minimum width over all $\theta \in[-\pi / 2, \pi / 2)$ that encloses $P$ can be computed in $O\left(n^{2}\right)$ time.

\subsection{General case}

Finally, we consider the general case where both $\theta$ and $\phi$ can be freely chosen from domain $[-\pi / 2, \pi / 2)$. Let $w^{*}:=\min _{\theta, \phi \in[-\pi / 2, \pi / 2)} w(\theta, \phi)$ be the minimum possible width, and $\left(\theta^{*}, \phi^{*}\right)$ be a pair of orientations such that $w^{*}=w\left(\theta^{*}, \phi^{*}\right)$.

We first consider the decision version of the problem in which a positive real number $w>0$ is given and we want to decide if $w \geq w^{*}$ or $w<w^{*}$. For the purpose, we consider the function $d_{p}$ defined above for each $p \in P$ to be $d_{p}(\theta)=\min \left\{\sigma_{\theta}\left(p, \chi^{+}(\theta)\right), \sigma_{\theta}\left(p, \chi^{-}(\theta)\right)\right\}$. As observed above, the function $d_{p}$ is piecewise sinusoidal with $O(n)$ breakpoints, so its graph $\left\{(\theta, y) \mid y=d_{p}(\theta),-\pi / 2 \leq \theta<\pi / 2\right\}$ consists of $O(n)$ sinusoidal curves. Let $\Gamma_{p}$ be the set of these sinusoidal curves, and $\Gamma:=\bigcup_{p \in P} \Gamma_{p}$. We build the arrangement $\mathcal{A}(\Gamma)$ of these sinusoidal curves in $\Gamma$. Note that each vertex of $\mathcal{A}(\Gamma)$ corresponds either to a breakpoint of function $d_{p}$ for some $p \in P$ or to an intersection point between a curve in $\Gamma_{p}$ and another in $\Gamma_{p^{\prime}}$ for some $p, p^{\prime} \in P$ with $p \neq p^{\prime}$.

- Lemma 20. The arrangement $\mathcal{A}(\Gamma)$ of curves in $\Gamma$ consists of $O\left(n^{3}\right)$ vertices, edges, and cells, and can be computed in $O\left(n^{3}\right)$ time.

Now, we describe our decision algorithm. Let $w>0$ be a given positive real number. First, we intersect the horizontal line $\ell:\{y=w\}$ with arrangement $\mathcal{A}(\Gamma)$.

- Lemma 21. Any horizontal line crosses the edges of $\mathcal{A}(\Gamma)$ in $O\left(n^{2}\right)$ points, and all these intersection points can be specified in $O\left(n^{2}\right)$ time.

Our algorithm continuously increases $\theta$ from $-\pi / 2$ to $\pi / 2$ and checks if there exists a parallelogram annulus of width $w$ that encloses $P$ such that one of the two double-strips defining it is $\theta$-aligned. 
Let $\left\{\theta_{1}, \ldots, \theta_{m}\right\}$ be the set of $\theta$-values of every intersection point between $\ell$ and the edges of $\mathcal{A}(\Gamma)$ such that $-\pi / 2 \leq \theta_{1}<\theta_{2}<\cdots<\theta_{m}<\pi / 2$. Note that $m=O\left(n^{2}\right)$ by Lemma 21 . For each $i=1, \ldots, m$, let $P_{i} \subseteq P$ be the set of points $p \in P$ such that $d_{p}\left(\theta_{i}\right) \leq w$, and let $Q_{i}:=P \backslash P_{i}$. Let $D_{i}$ be the $\theta_{i}$-aligned $P$-constrained double-strip of width $w$. Then, we have $P_{i} \subset D_{i}$ while $Q_{i} \cap D_{i}=\emptyset$. Let $D_{i}^{\prime}$ be a $P$-constrained double-strip of minimum width that encloses $Q_{i}$. Recall that the width of $D_{i}^{\prime}$ is denoted by $w_{Q_{i}}^{*}$ in the previous section. If the width $w_{Q_{i}}^{*}$ of $D_{i}^{\prime}$ is at most $w$, then the parallelogram annulus defined by $D_{i}$ and $D_{i}^{\prime}$ indeed encloses $P$ and its width is $w$, so we conclude that $w \geq w^{*}$. Otherwise, if $w_{Q_{i}}^{*}>w$, then we proceed to next $\theta$-value $\theta_{i+1}$.

We perform this test for each $i=1, \ldots, m$ in an efficient way with the aid of our online decision algorithm for the constrained double-strip problem. Initially, for $i=1$, we compute $P_{1}, Q_{1}$, and $D_{1}$ in $O(n)$ time. Also, we initialize the data structures $\mathcal{T}_{\emptyset}$ and fixed value $w$, as described in Theorem 14 in $O(n \log n)$ time, and insert points in $Q_{1}$ to have $\mathcal{T}_{Q_{1}}$ in $O\left(\left|Q_{1}\right| n\right)=O\left(n^{2}\right)$ time. We then know that $w_{Q_{1}}^{*} \geq w$ or not.

For each $i \geq 2$, there is a point $p \in P$ such that either $P_{i}=P_{i-1} \backslash\{p\}$ or $P_{i}=P_{i-1} \cup\{p\}$. Since $Q_{i}=P \backslash P_{i}$, we have that either $Q_{i}=Q_{i-1} \cup\{p\}$ or $Q_{i}=Q_{i-1} \backslash\{p\}$. This implies that we can answer whether $w \geq w_{Q_{i}}^{*}$ or not in $O(n)$ time for each $i \geq 2$ by maintaining $P_{i}$, $Q_{i}$, and $\mathcal{T}_{Q_{i}}$ by Theorem 14 .

Since $m=O\left(n^{2}\right)$ by Lemma 21, we can solve the decision problem in $O\left(n^{3}\right)$ time.

- Lemma 22. Given $w>0$, we can decide whether or not $w \geq w^{*}$ in $O\left(n^{3}\right)$ time.

Finally, we describe our algorithm to compute the exact value of $w^{*}$. To do so, we collect a set $W$ of candidate width values in which $w^{*}$ is guaranteed to exist, and perform a binary search using our decision algorithm summarized in Lemma 22.

- Lemma 23. The minimum possible width $w^{*}$ over all parallelogram annulli that enclose $P$ is equal to the $y$-coordinate of a vertex of $\mathcal{A}(\Gamma)$.

We thus define $W$ to be the set of $y$-coordinates of all vertices of $\mathcal{A}(\Gamma)$. Lemma 23 guarantees that $w^{*} \in W$. After sorting the values in $W$, we perform a binary search on $W$ using the decision algorithm. Since $|W|=O\left(n^{3}\right)$ by Lemma 20 and the decision algorithm runs in $O\left(n^{3}\right)$ time by Lemma 22, we can find the exact value of $w^{*}$ in $O\left(n^{3} \log n\right)$ time. Therefore, we conclude the following theorem.

Theorem 24. Given a set $P$ of $n$ points, a minimum-with parallelogram annulus over all pairs of orientations that encloses $P$ can be computed in $O\left(n^{3} \log n\right)$ time.

\section{References}

1 M. Abellanas, Ferran Hurtado, C. Icking, L. Ma, B. Palop, and P.A. Ramos. Best Fitting Rectangles. In Proc. Euro. Workshop Comput. Geom. (EuroCG 2003), 2003.

2 P. Agarwal and M. Sharir. Planar geometric location problems. Algorithimca, 11:185-195, 1994.

3 P.K. Agarwal and M. Sharir. Efficient randomized algorithms for some geometric optimization problems. Discrete Comput. Geom., 16:317-337, 1996.

4 P.K. Agarwal, M. Sharir, and S. Toledo. Applications of parametric searching in geometric optimization. J. Algo., 17:292-318, 1994.

5 Sang Won Bae. Computing a Minimum-Width Square Annulus in Arbitrary Orientation. Theoret. Comput. Sci., 718:2-13, 2018.

6 Sang Won Bae. On the Minimum-Area Rectangular and Square Annulus Problem. CoRR, 2019. arXiv: 1904.06832 . 
7 Mark de Berg, Mark van Kreveld, Mark Overmars, and Otfried Schwarzkopf. Computationsl Geometry: Alogorithms and Applications. Springer-Verlag, 2nd edition, 2000.

8 Alex Glozman, Klara Kedem, and Gregory Shpitalnik. On some geometric selection and optimization problems via sorted matrices. Comput. Geom.: Theory Appl., 11(1):17-28, 1998.

9 Olga N. Gluchshenko, Horst W. Hamacher, and Arie Tamir. An optimal $O(n \log n)$ algorithm for finding an enclosing planar rectilinear annulus of minimum width. Operations Research Lett., 37(3):168-170, 2009.

10 J. Hershberger. Finding the upper envelope of $n$ line segments in $O(n \log n)$ time. Inform. Proc. Lett., 33:169-174, 1989.

11 Jerzy Jaromczyk and Miroslaw Kowaluk. The Two-Line Center Problem from a Polar View: A New Algorithm and Data Structure. In Proc. 4th Int. Workshop Algo. Data Struct. (WADS 1995), volume 955 of Lecture Notes Comput. Sci., pages 13-25, 1995.

12 J. Mukherjee, P.R.S. Mahapatra, A. Karmakar, and S. Das. Minimum-width rectangular annulus. Theoretical Comput. Sci., 508:74-80, 2013.

13 U. Roy and X. Zhang. Establishment of a pair of concentric circles with the minimum radial separation for assessing roundness error. Computer-Aided Design, 24(3):161-168, 1992.

14 G.T. Toussaint. Solving geometric problems with the rotating calipers. In Proc. IEEE MELECON, 1983. 